\title{
Robust Image Watermarking using Fractional Sinc Transformation
}

\author{
Almas Abbasi \\ Department of Computer Science and software engineering \\ International Islamic University \\ 44000 Islamabad, Pakistan
}

\author{
Chaw Seng Woo \\ Faculty of Computer Science and Information Technology \\ Department of Artificial Intelligence \\ University of Malaya \\ 50603 Kuala Lumpur, Malaysia
}

\begin{abstract}
The increased utilization of internet in sharing and dissemination of digital data makes it is very difficult to maintain copyright and ownership of data. Digital watermarking offers a method for authentication and copyright protection. Digital image watermarking is an important technique for the multimedia content authentication and copyright protection. This paper present a watermarking algorithm making a balance between imperceptibility and robustness based on fractional calculus and also a domain has constructed using fractional Sinc function (FSc). The FSc model the signal as polynomial for watermark embedding. Watermark is embedded in all the coefficients of the image. Cross correlation method based on Neyman-Pearson is used for watermark detection. Moreover fraction rotation expression has constructed to achieve rotation. Experimental results confirmed the proposed technique has good robustness and outperformed another technique in imperceptibility. Furthermore the proposed method enables blind watermark detection where the original image is not required during the watermark detection and thus making it more practical than non-blind watermarking techniques.
\end{abstract}

Keywords-Fractional Calculus; fractional Sinc; image Watermarking; robust

\section{INTRODUCTION}

Receiving and transmitting digital data has instigated its wide appearance and storage. The use of digital data on a broader scale has brought a lot of ease in different aspects, but it is not without side effects such as tempering and copyright protection issues. With digital data so widely used, watermarking are mostly used to address these issues. Watermarking is a method to embed a message while stenography is the art of hidden communication. The purpose of watermarking is to keep the message secret whereas data hiding is general term and covers a vast range of problems related to making information confidential.

In general watermarking techniques necessitates certain properties. These properties are required for all kinds of multimedia data such as audio, video and images. However, the significance of these properties varies with the purpose and application of watermarking. In case of copyright protection application the watermark should be robust enough to resist any attempt for its removal. While for the authentication applications, robustness is not required. The Fundamental watermarking system properties are: Imperceptibility and robustness.
Watermarking schemes are usually based on special domain methods [1, 2, 3] as well as transformed domain techniques such as DCT, DFT, and wavelet, etc. Watermarking in spatial domain is straight forward and easy to implement as compared to watermarking in transformed [4, 5, 6] domain. Historically spatial domain watermarking was the first watermarking scheme the researcher had investigated upon. However, it has low robustness compared to transformation domain as the watermark easy be obliterated by lossy image compression techniques. Most common example of spatial domain method is Least significant bit (LSB). The watermark embedded in the least bit is least perceive by human eye. However the spatial domain methods are not robust i-e. They cannot survive various kinds of attacks as the least bit are usually removed or destroyed in these attacks. Transform domain methods embed the watermark into the transformed coefficients of the original image. Transform based methods are most popular as they are generally more robust to malicious attacks. In transform domain method the transformed coefficient of the original image is altered to embed watermark. Researchers have considered many image transformations. Most popular transform domains are discrete cosine transformation (DCT), discrete wavelet transforms (DWT), and discrete Fourier transforms (DFT). Moreover, polynomial transformation based watermarking techniques are proposed by researchers to obtain improved imperceptibility and robustness. Researchers have also used polynomial based transformation for watermarking [7-9].

Most of the above mentioned techniques embed watermark in selected areas to get robust watermarking. In the proposed technique watermark is embedded in the whole image while in order to achieve balance between imperceptibility and robustness new model based on Fractional order of Sinc is used.

\section{FRACTIONAL CALCULUS}

Fractional calculus is a mathematical discipline which deals with derivatives and integrals of arbitrary real or complex orders. The history of fractional calculus is 300 years old and genuine expansion and progression has seen in $19^{\text {th }}$ century. During the last decade fractional calculus has been applied to different fields most noticeable among them are physics, biology, science, engineering, image processing and other fields. Fractional Calculus generalized the ideas of integer 
order differentiation and n-fold integration. Fractional order differentiators and integrators are example of fractional order systems. Fractional order systems are defined by fractional order differential equations. Fractional order differentiators and integrators are used to compute the fractional order time derivative and integral of the given signal [10-15]. Fractional derivatives introduce an excellent instrument for the description of general properties of various materials and processes such as signal processing and image processing [16]. One of the advantages of fractional calculus is that it can be well thought-out as a super set of integer order calculus. Thus fractional calculus has the potential to realize what the integer order calculus cannot. One of these famous operators is the Riemann-Liouville operator (differential and integral), which defined in definition 2.1

\section{A. The Sinc function and its properties}

1. The limit of sinc:

$\lim _{x \rightarrow 0} \frac{\sin x}{x}=1$.

i-e $\lim$ of $\operatorname{sinc}(x)$ approaches to Heaviside function.

2. Expression for universal constant $\pi$ :

$$
\sin c(x)=\left(1-\frac{x^{2}}{1^{2} \pi^{2}}\right)\left(1-\frac{x^{2}}{2^{2} \pi^{2}}\right)\left(1-\frac{x^{2}}{3^{2} \pi^{2}}\right) \cdots \cdots \cdots \oplus^{\alpha} t^{\mu}=\frac{\Gamma(\mu+1)}{\Gamma(\mu-\alpha+1)} t^{\mu-\alpha}, \mu>-1 ; 0<\alpha<1
$$

3. Fourier analysis: The sinc function appears frequently in Fourier analysis. This appearance of sinc explains its occurrence in areas of engineering that rely on the analysis of signals such as communication theory. Fourier analysis allow us to expand the function in a trigonometric series of the form:

$$
f(x)=a_{0}+a_{1} \cos x+b_{1} \sin x+a_{2} \cos 2 x+b_{2} \sin 2 x+\ldots \ldots \ldots
$$

$$
I^{\alpha} t^{\mu}=\frac{\Gamma(\mu+1)}{\Gamma(\mu+\alpha+1)} t^{\mu+\alpha}, \mu>-1 ; \alpha>0 \text {. }
$$

This paper investigate the utilization of the Sinc basis functions

Definition 2.3. The function $\operatorname{Sinc}(t)$ defined by

$$
\operatorname{Sinc}(t)=\frac{\sin (t)}{t}, \quad \mathrm{t} \neq 0
$$

Where $a_{i}$ and the $b_{i}$ are constants.

4. Shannon entropy: for a function $f$, one can interpolate it as follows:

$$
C(x)=\sum_{n=-\infty}^{\infty} f(n h) \sin c \pi\left(\frac{x}{h}-n\right) \text {. }
$$

Where $n=0, \pm 1, \pm 2, \pm 3, \ldots \ldots \ldots . .=0$, and $h>0$. [17]

\section{Jordan's inequality:}

$$
|\sin c(x)| \leq 1 \text {. }
$$

This lead to all the derivations of $\operatorname{sinc}(\mathrm{x})$ are also less than 1.
For fractional $\operatorname{Sinc}(t)$ of order $\alpha,\left(S c_{\alpha}\right)$, defined by [10].

$$
\begin{aligned}
S c_{\alpha}(t) & =\frac{\sin _{\alpha}(t)}{t} \\
= & \sum_{n=0}^{\infty} \frac{(-1)^{n} t^{(2-\alpha) n+1}}{\Gamma((2-\alpha) n+2)}, \\
\qquad & =s+\left(w_{b} \times \kappa\right)
\end{aligned}
$$


Where

$$
\sin _{\alpha}(t)=\sum_{n=0}^{\infty} \frac{t^{n-\alpha}}{\Gamma(n-\alpha+1)} \sin \left((n-\alpha) \frac{\pi}{2}\right), \quad \Gamma
$$

is the gamma function, $t$ is the variable and $\alpha \in(0,1)$ is a constant. From Definition 2.3, the following coefficients are obtained

$$
\begin{aligned}
& \phi_{0}=\frac{1}{\Gamma(2)} \\
& \phi_{1}=\frac{(-1)}{\Gamma(4-\alpha)} \\
& \phi_{2}=\frac{1}{\Gamma(6-2 \alpha))} \\
& \vdots \\
& \phi_{\mathrm{n}}=\frac{(-1)^{n}}{\Gamma\left((2-\alpha)^{n}+2\right)}
\end{aligned}
$$

\section{PROPOSED TECHNIQUE}

The watermarking domain used in this technique is transform domain based on FSc. The watermark embedding and extraction is performed in FSc domain. Further the watermarking technique used in this study is blind watermarking technique. In blind watermarking technique original image is not required for the watermark detection and is more practical than non-blind watermarking techniques.

\section{A. Balanced watermarking}

The basic requirement of a Watermarking technique is to achieve high imperceptibility and robustness. These two properties are inverse to each other. When the imperceptibility increase, robustness decrease and vice versa. A balance between imperceptibility and robustness is required for a watermarking technique. In this technique Fractional calculus transformation has been used to achieve the balance.

\section{B. Watermark embedding}

Let $I(i, j)$ represent the original gray scale image of size $\mathrm{M} \times \mathrm{N}$ pixels and $\mathrm{w}(\mathrm{i}, \mathrm{j})$ is the watermark pattern to be embedded using additive embedding technique. Generally, additive embedding is implemented by using I' $=\mathrm{I}+\mathrm{k} w$, where I' is the watermarked image, and $\mathrm{k}$ is the embedding strength. The size of the watermark $w$ is equal to the size of the image selected for watermark embedding. The strength of watermark $\mathrm{k}$ is kept as a constant value.

For the current implementation, the watermark signal consists of $\{+1,-1\}$ bits. The watermark embedding process can be represented by the following equation:

where $\mathrm{S}$ represents the watermark signal, $\mathrm{s}$ is the original signal (i.e. coefficients in our case), $w_{\mathrm{b}}$ is the watermark bit. The parameter $\kappa$ is a constant value.
The proposed watermarking embedding technique includes the following steps:

- Read the grayscale images of size $512 \times 512$.

- Apply fractional Sc $\alpha$ transformation:

a. Set the parameter $\alpha>0$.

b. Fix the value of the variable $t$.

c. Calculate the fractional order of Sc $\alpha$ polynomial using Definition 2.3

- Embed the watermark using the Eq. (3).

- Calculate inverse fractional $\mathrm{Sc} \alpha$ by taking transpose of the resultant image.

- Perform above steps for each image.

\section{Watermark detection}

Watermark detection is in fact the reverse of the embedding processes. Watermarked image transformed using FSc. The correlation between the watermarked coefficient and the watermark to be tested for the existence is computed using the following expression:

$$
\rho=\frac{1}{M N} \sum_{i=0}^{M-1} \sum_{j=0}^{N-1} I_{0}^{\prime}(i, j) w(i, j)
$$

where $I_{0}^{\prime}(i, j)$ is the FSc coefficient and $w(i, j)$ represents the watermark. $M N$ is the size of the image. The computed value of $\rho$ is then compared to the threshold value $T_{\rho}$ calculated as follows:

$$
T_{\rho}=3.97 \sqrt{2 \sigma_{\rho \beta}^{2}}
$$

where $\sigma_{\rho \beta}^{2}$ represents the variance and

$$
\sigma_{\rho \beta}^{2} \approx \frac{1}{(M N)^{2}} \sum_{i=0}^{M-1} \sum_{j=0}^{N-1}\left(I_{0}^{\prime}(i, j)\right)^{2}
$$

For the detailed derivation of these equations, please refer the interested readers to [6]. The existence of the watermark will be confirmed when $\rho>T p$.

The proposed technique watermark extraction steps are summarize in the following pseudo code:

- Read the watermark/attacked image.

- Apply FSc transformation on the image.

- Compute $\rho$ and $T_{\rho}$ using equation 4 and 5 respectively.

- Compare $\rho$ and $T_{\rho}$, If $\rho>T_{\rho}$ then watermark is detected otherwise not.

- Repeat above steps for all images. 


\section{EXPERIMENTS ANALYSIS}

In this section, the performance of the proposed watermarking technique has evaluated by considering robustness and imperceptibility. Five standard test images from the USC-SIPI dataset [18], namely, Baboon, Cameraman, Lena, Peppers, and Sailboat, are considered for evaluation purposes. These images are each of dimensions $512 \times 512$. As a proof of concept, the algorithm is coded by using Matlab and checkmark software is deployed for testing the robustness against different set of attacks. The watermark signal is presented by the sequence of +1 or -1 .

TABLE I. PSNR, MSE AND SSIM VALUE OF SAMPLE TEST IMAGES IN THE PROPOSED FSC DOMAIN

\begin{tabular}{|l|l|l|l|l|l|}
\hline & Lena & Baboon & Cameraman & $\begin{array}{l}\text { Sailboat } \\
\text { Boat }\end{array}$ & Peppers \\
\hline PSNR & 42.02 & 41.80 & 42.52 & 41.54 & 41.37 \\
\hline MSE & 4.09 & 4.30 & 3.64 & 4.56 & 4.74 \\
\hline SSIM & 0.93 & 0.98 & 0.92 & 0.95 & 0.94 \\
\hline
\end{tabular}

\section{A. Imperceptibility}

The watermarked images obtained by using the proposed technique where total 262144 bits of watermark is embedded. By visual inspection, the watermarked images appear perceptually similar to their original counterparts. To quantify the transparency of the embedded watermark, the peak signal to noise ratio (PSNR) and structure similarity index measure (SSIM) are considered, which are commonly used by the watermarked community. The results are recorded in Table 1. It is observed that the PSNR and SSIM values range from 41.37 to $42.52 \mathrm{~dB}$ and 0.92 to 0.98 , respectively. These readings suggest that the watermark image generated by the proposed method is of high perceptual quality.

\section{B. Robustness}

Fig 1(a-e) summaries the different set of attacks performed for the evaluation of our proposed watermarking technique. Cross correlation method based on Neyman-Pearson is deployed to detect the embedded watermark and its formula is detailed in [6].

In our experiment, the watermarked images have gone various types of attack to investigate the robustness of the proposed technique. In particular, each watermarked image is distorted using different geometric and image processing attacks, namely: (1) rescaling attack with scaling factor ranging from 0.5 to 2; (2) JPEG compression with quality factor ranging from $50 \%$ to $90 \%$ with increment of 10 ; (3) row and column removal attack with the number of rows and column removal varying from 1 to 17 ; (4) change in aspect ratio in the $\mathrm{x}$ and $\mathrm{y}$ directions; (5) Gaussian filtering with kernel size of $3 \times 3$ and $4 \times 4$ pixels; (6) sharpening attack. (7) Cropping attack with 10,20 and $50 \%$ cropped relative to the size of image. Fig 3.(a- t) shows the result of applying these attacks.

The cross correlation computation based on NeymanPearson criterion and the threshold value are considered to test the presence of the embedded watermark.

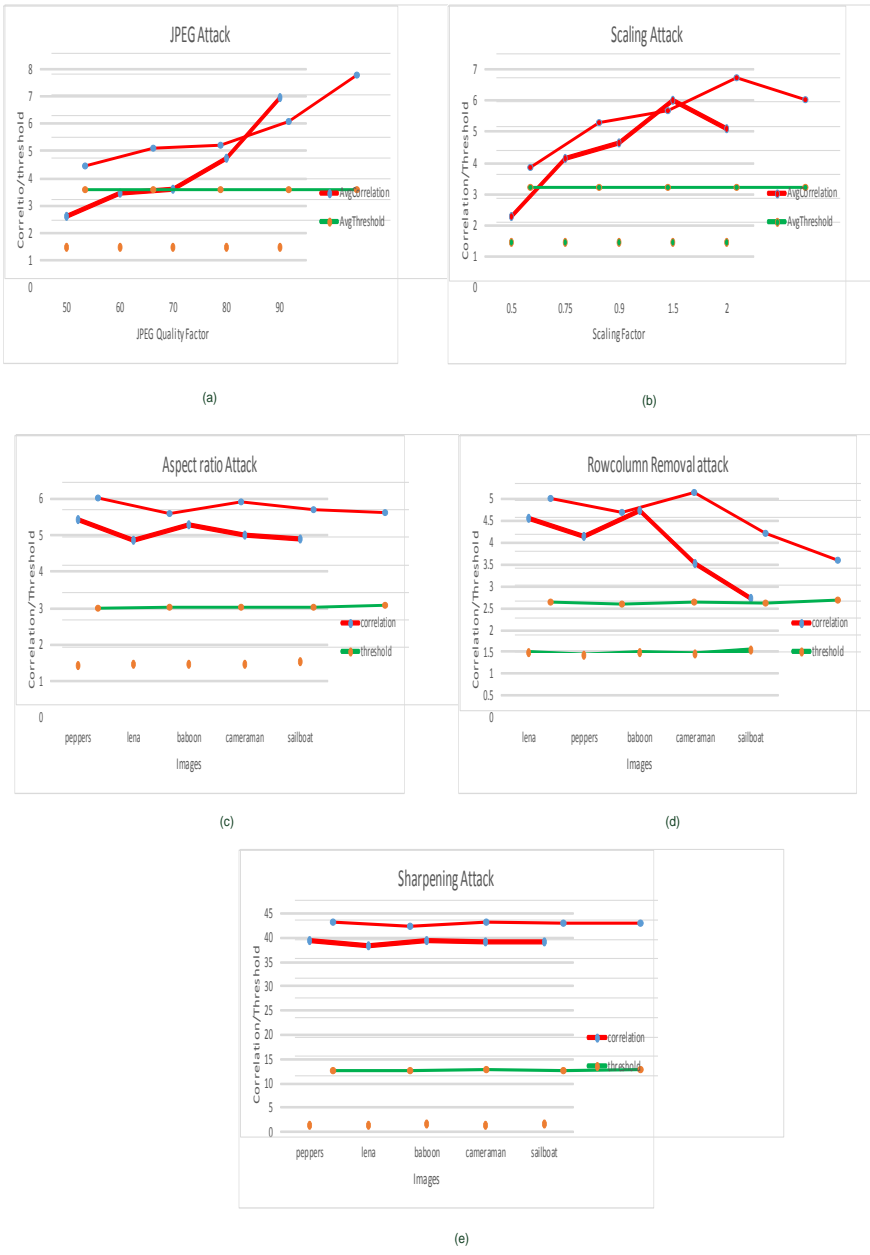

Fig. 1. Robustness of Proposed Technique against different types of attacks. (a) Comparison of average correlation values of five standard images after JPEG compression attack with quality factors changes from 50 to 90 , against dynamic threshold. (b) Comparison of average correlation values of five standard images after scaling attack of different scaling factors i-e from 0.5 to 2, against dynamic threshold. (c) Aspect ratio attack of 2:7 in relation to $\mathrm{x}$ and y axis. (d) Row Column removal attack $(17,5)$. (e) Sharpening attack
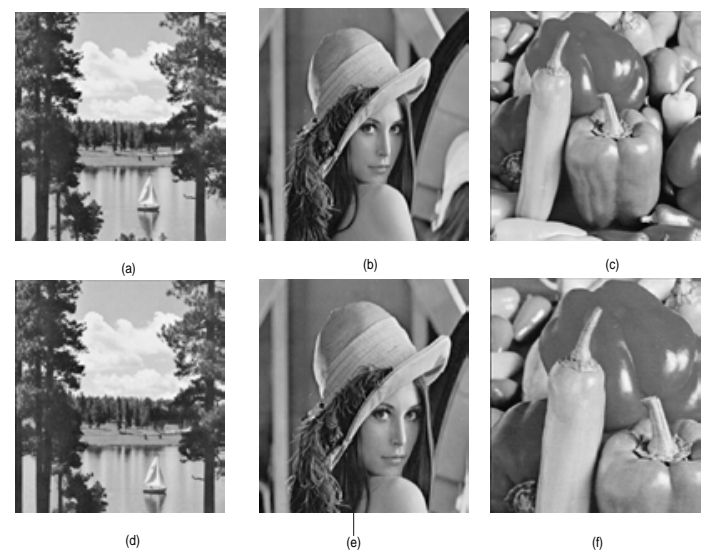

Fig. 2. (a-c) Original test images, (d-f) Attacked watermark images for different cropped size such as $10 \%, 20 \%$, and $50 \%$ of the image size respectively 
The scaling attack using various factors ranging from 0.5 to 2.0, remains well above the (i.e., threshold) considered as suggested by Fig. 1(b). Robustness against JPEG compression with quality factor ranging from $50 \%$ to $90 \%$ are shown in Fig 1(a). The presence of watermark is detected in all these cases, as the Correlation values lies quite above the threshold value. Moreover, common image processing operations such as, aspect ratio change Fig 1(c), row and column removal attack Fig 1(d), sharpening and Gaussian filtering Fig 1 (e) are also applied on the watermarked image. For all cases, the dynamically computed value always stays above the threshold , i.e., $100 \%$ successful detection. Therefore, the results suggest that the proposed method is robust against the commonly considered watermarking distortion attacks. Fig 3 shows the images after the above mentioned different set of attacks.

Fig 2. Represent cropping attack performed on the watermark image to test the robustness against different size of cropped attempt. Fig 2(a-c) represent original test images while Fig 2(d-f) represent the cropping using 10\%, 20\%, and 50\% of image size. This shows that the proposed watermarking scheme is robust against the cropping attack.

\section{FRACTIONAL MATRIX EXPRESSION FOR ROTATION}

A new rotation expression has derived using fractional trigonometric functions. Fractional rotation is a special case of fractional sinc. Now let us examine rotation of images by utilizing method based on the fractional derivative $\mathrm{D} \alpha$ of $\sin (\mathrm{t}$ ) and $\cos (\mathrm{t})$. The following result are obtained.

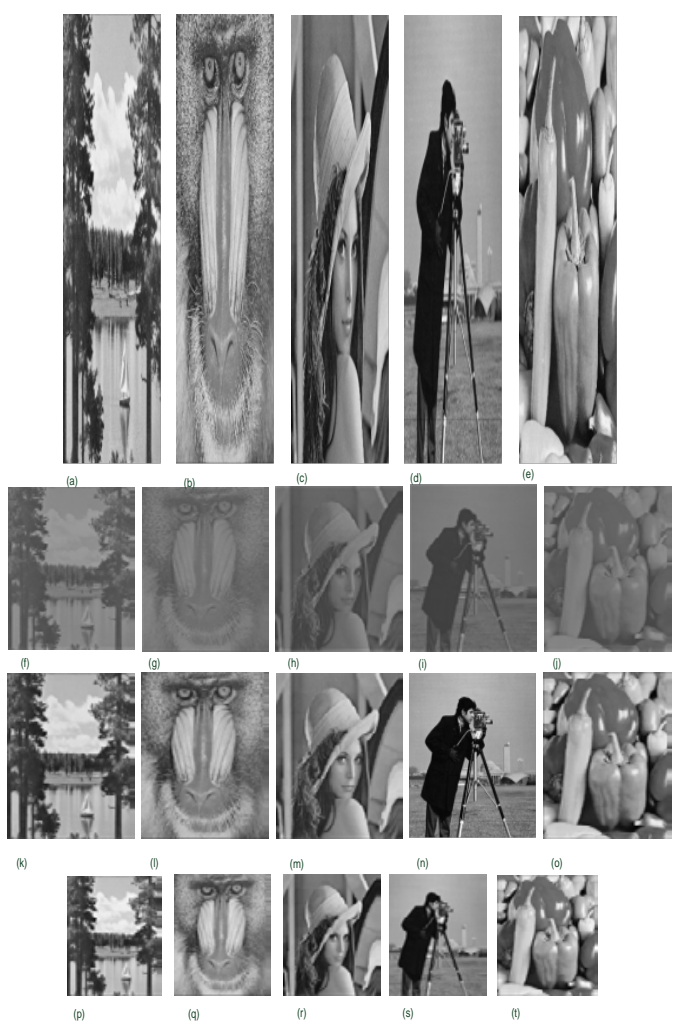

Fig. 3. Experimental results:(a) to (e) show the watermarked test images manipulated by using aspect ratio of 2:7 in relation to $\mathrm{x}$ and $\mathrm{y}$ axis; (f) to (j) represent watermarked images corrupted by using sharpening attack;(k) to (o) illustrate the watermarked images are compressed with JPEG compression attack with quality factor $50 ;(\mathrm{p})$ to (t) display the scaled down to $50 \%$ of the image size

Lemma 5.1 Let $\cos _{\alpha}(t)$ and $\sin _{\alpha}(t)$ defined respectively by

$$
\cos _{\alpha}(t)=\sum_{n=0}^{\infty} \frac{t^{n-\alpha}}{\Gamma(n-\alpha+1)} \cos \left((n-\alpha) \frac{\pi}{2}\right)
$$

And

$$
\sin _{\alpha}(t)=\sum_{n=0}^{\infty} \frac{t^{n-\alpha}}{\Gamma(n-\alpha+1)} \sin \left((n-\alpha) \frac{\pi}{2}\right)
$$

The fractional rotation obtained are:

$$
\mathrm{R}_{\alpha}=\left(\begin{array}{cc}
\cos (\alpha \pi / 2) & \sin (\alpha \pi / 2) \\
-\sin (\alpha \pi / 2) & \cos (\alpha \pi / 2)
\end{array}\right)
$$

Proof. By using Definition 2.2 together with Remark 2.1, we obtain that

$$
\begin{aligned}
& D^{\alpha} \sin (t)=\sin (\alpha \pi / 2) \cos _{\alpha}(\mathrm{t})+\cos (\alpha \pi / 2) \sin _{\alpha}(\mathrm{t}) \\
& \text { and } \\
& D^{\alpha} \cos (\mathrm{t})=\cos (\alpha \pi / 2) \cos _{\alpha}(\mathrm{t})-\sin (\alpha \pi / 2) \sin _{\alpha}(\mathrm{t})
\end{aligned}
$$

A geometrical interpretation of the derivative relations in Eq 9 and Eq 10 can be found by imposing the matrix form

$$
\mathrm{R}_{\mathrm{D}^{\alpha}}=\left(\mathrm{D}^{\alpha} \cos (t) \quad \mathrm{D}^{\alpha} \sin (t)\right)
$$

Collecting the coefficients of $\mathrm{R}_{\mathrm{D}^{\alpha}}$, the following fractional rotation matrix obtained:

$$
\mathrm{R}_{\alpha}=\left(\begin{array}{cc}
\cos (\alpha \pi / 2) & \sin (\alpha \pi / 2) \\
-\sin (\alpha \pi / 2) & \cos (\alpha \pi / 2)
\end{array}\right)
$$

By applying rotation (8) of different degrees such as 5, 10, 15, 20, 25, 30, 35, 40 to the proposed scheme achieve fractional rotation. Result are shown in Fig 4.

Remark 5.2 One can use the transpose of $\mathrm{R}_{\mathrm{D}^{\alpha}}$ to get good result as well.

$$
\mathrm{R}_{\alpha}{ }^{T}=\left(\begin{array}{ll}
\cos (\alpha \pi / 2) & -\sin (\alpha \pi / 2) \\
\sin (\alpha \pi / 2) & \cos (\alpha \pi / 2)
\end{array}\right)
$$




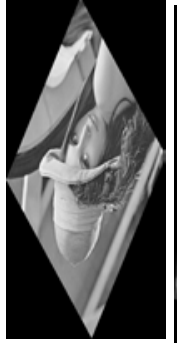

(a)

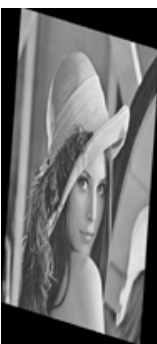

(b)

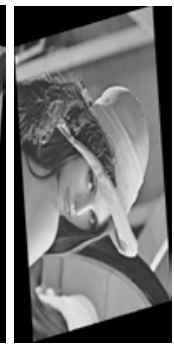

(c)

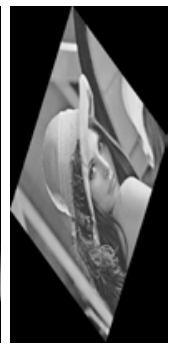

(d)
Fig. 4. (a-d) Rotation achieved using fractional rotation expression having angle $15,25,30$ and 45 respectively

\section{CONCLUSION}

Digital image watermarking is an important technique for the multimedia content authentication and copyright protection. Some watermarking techniques are extremely robust but they suffer poor imperceptibility. This paper introduce a watermarking algorithm balanced between imperceptibility and robustness based on fractional calculus. A new domain has been constructed using fractional order of Sinc (FSc). Moreover fraction rotation expression has been designed to achieve rotation. The FSC model the signal as a fractional polynomial for watermark embedding. Watermark is embedded in all the coefficients of the image. Cross correlation method based on Neyman-Pearson is used for watermark detection. Experimental results confirmed the proposed technique is robust and imperceptible.

\section{ACKNOWLEDGMENT}

Authors would like to thank all those who provide technical help and correction in writing this article.

\section{REFERENCES}

[1] Nikolaidis, N. and Pitas, I. , 'Robust image watermarking in the spatial domain', Signal processing, 66, pp.385-403,1998.

[2] Darmstaedter, V., Delaigle, J.-F., Nicholson, D. and Macq, B., 'A block based watermarking technique for MPEG2 signals: Optimization and validation on real digital TV distribution links', in Multimedia Applications, Services and Techniques-ECMAST'98: Springer, pp. 190-206,1998.
[3] Wolfgang, R.B. and Delp, E.J., 'A watermark for digital images', in Image Processing, 1996. Proceedings. International Conference on: IEEE, pp. 219-222, 1996

[4] Hernandez, J.R., Amado, M. and Perez-Gonzalez, F., 'DCT-domain watermarking techniques for still images: Detector performance analysis and a new structure', Image Processing, IEEE Transactions on, 9, pp.5568,2000 .

[5] Kundur, D. and Hatzinakos, D., 'Digital watermarking using multiresolution wavelet decomposition', in Acoustics, Speech and Signal Processing, 1998. Proceedings of the 1998 IEEE International Conference on: IEEE, pp. 2969-2972,1998.

[6] Barni, M., Bartolini, F. and Piva, A., 'Improved wavelet-based watermarking through pixel-wise masking', Image Processing, IEEE Transactions on, 10, pp.783-791,2001.

[7] Le, L., Krishnan, S. and Ghoraani, B., 'Discrete Polynomial Transform for Digital Imagewatermarking Application', in Multimedia and Expo, 2006 IEEE International Conference on, pp. 1569-1572,2006.

[8] Krishnamoorthi, R.M., P. D. Sheba Kezia., 'Image Adaptive Watermarking with Visual Model in Orthogonal Polynomials based Transformation Domain', International Journal of Signal Processing;2009, Vol. 5 Issue 2, pp.146, April,2009.

[9] Al-Qaheri, H., Mustafi, A. and Banerjee, S. , 'Digital watermarking using ant colony optimization in fractional Fourier domain', Journal of Information Hiding and Multimedia Signal Processing, 1, pp.179$189,2010$.

[10] Podlubny, I., Fractional Differential Equations: Academic Press, San Diego - New York - London,1999.

[11] K.B. Oldham, J. Spanier, The Fractional Calculus, Academic Press, New York, 1974.

[12] K.S. Miller, B. Ross, An Introduction to the Fractional Calculus and Fractional Differential Equations, John Wiley Sons, 1993.

[13] B.J. west, M. Bologna, P. Grigolini, Physics of Fractal Operators, Springer Verlag, 2003.

[14] Tofighi, A. and Pour, H.N., 'e-expansion and the fractional oscillator', Physica A: Statistical Mechanics and its Applications, 374, pp.41$45,2007$.

[15] Ortigueira, M.D., 'An introduction to the fractional continuous-time linear systems',2008.

[16] Abbasi A, Woo CS, Ibrahim RW, Islam S Invariant Domain Watermarking Using Heaviside Function of Order Alpha and Fractional $\begin{array}{llll}\text { Gaussian Field. PLoS ONE 10(4): e0123427. } & \end{array}$ doi:10.1371/journal.pone.0123427,2015.

[17] Gearhart, W. B., \& Schultz, H. S. , The function sin (x) x. The College Mathematics Journal, 2,pp. 90-99,1990.

[18] USC-SIPI. (2014). USC-SIPI image database. Online:http://sipi.usc.edu/database/. 